\title{
Vertex Degrees and Isomorphic Properties in Complement of an $m$-Polar Fuzzy Graph
}

\author{
Ch. Ramprasad, ${ }^{1,2}$ P. L. N. Varma, ${ }^{2}$ S. Satyanarayana, ${ }^{3}$ and N. Srinivasarao ${ }^{4}$ \\ ${ }^{1}$ Department of Mathematics, Vasireddy Venkatadri Institute of Technology, Nambur 522 508, India \\ ${ }^{2}$ Department of Mathematics, VFSTR University, Vadlamudi 522 237, India \\ ${ }^{3}$ Department of CSE, KL University, Vaddeswaram 522502, India \\ ${ }^{4}$ Department of Mathematics, Tirumala Engineering College, Narasaraopet 522034, India
}

Correspondence should be addressed to S. Satyanarayana; s.satyans1@gmail.com

Received 22 May 2017; Accepted 5 July 2017; Published 22 August 2017

Academic Editor: Mehmet Onder Efe

Copyright (c) $2017 \mathrm{Ch}$. Ramprasad et al. This is an open access article distributed under the Creative Commons Attribution License, which permits unrestricted use, distribution, and reproduction in any medium, provided the original work is properly cited.

Computational intelligence and computer science rely on graph theory to solve combinatorial problems. Normal product and tensor product of an $m$-polar fuzzy graph have been introduced in this article. Degrees of vertices in various product graphs, like Cartesian product, composition, tensor product, and normal product, have been computed. Complement and $\mu$-complement of an $m$-polar fuzzy graph are defined and some properties are studied. An application of an $m$-polar fuzzy graph is also presented in this article.

\section{Introduction}

Akram [1] introduced the notion of bipolar fuzzy graphs describing various methods of their construction as well as investigating some of their important properties. Bhutani [2] discussed automorphism of fuzzy graphs. Chen et al. [3] generalized the concept of bipolar fuzzy set to obtain the notion of an $m$-polar fuzzy set. The notion of an $m$-polar fuzzy set is more advanced than fuzzy set and eliminates ambiguity more absolutely. Ghorai and Pal [4] studied some operations and properties of an $m$-polar fuzzy graph. Mordeson and Peng [5] defined join, union, Cartesian product, and composition of two fuzzy graphs. Rashmanlou et al. [6] discussed some properties of bipolar fuzzy graphs and their results. Sunitha and Vijaya Kumar [7] defined the complement of a fuzzy graph in another way which gives a better understanding about that concept. We have studied product $m$-polar fuzzy graph, product $m$-polar fuzzy intersection graph, and product $m$-polar fuzzy line graph [8].

In this article, we study the Cartesian product and composition of two $m$-polar fuzzy graphs and compute the degrees of the vertices in these graphs. The notions of normal product and tensor product of $m$-polar fuzzy graphs are introduced and some properties are studied. Also in the present work, we introduce the concept of complement, $\mu$ complement of an $m$-polar fuzzy graph, and some properties are discussed.

These concepts strengthen the decision-making in critical situations. Some applications to decision-making are also studied.

In this article, unless and otherwise specified, all graphs considered are $m$-polar fuzzy graphs.

\section{Preliminaries}

Definition 1. The $m$-polar fuzzy graph of a graph $G^{*}=(V, E)$ is a pair $G=(W, F)$, where $W: V \rightarrow[0,1]^{m}$ is an $m$ polar fuzzy set in $V$ and $F: V \widetilde{\times} V \rightarrow[0,1]^{m}$ is an $m$-polar fuzzy set in $V \tilde{\times} V$ such that $F(q r) \leq \min \{W(q), W(r)\}$ for all $q r \in V \widetilde{\times} V$ and $F(q r)=0$ for all edges $q r \in\left(\widetilde{V}^{2}-E\right)(0=$ $0,0, \ldots, 0)$ is the smallest element in $[0,1]^{m} . W$ is called the $m$-polar fuzzy vertex set of $G$ and $F$ is called $m$-polar fuzzy edge set of $G$.

Sometimes we denote the graph $G=(W, F)$ by $G=$ $(V, W, F)$ also.

Definition 2. Given two graphs $G_{1}, G_{2}$ their Cartesian product, $G_{1} \times G_{2}=\left(V_{1} \times V_{2}, W_{1} \times W_{2}, F_{1} \times F_{2}\right)$, is defined as follows: 
for $i=1,2, \ldots, m$, we have

(i) $p_{i} \circ\left(W_{1} \times W_{2}\right)\left(q_{1}, q_{2}\right)=\min \left\{p_{i} \circ W_{1}\left(q_{1}\right), p_{i} \circ W_{2}\left(q_{2}\right)\right\}$ for all $\left(q_{1}, q_{2}\right) \in V_{1} \times V_{2}$,

(ii) $p_{i} \circ\left(F_{1} \times F_{2}\right)\left(\left(q, q_{2}\right)\left(q, r_{2}\right)\right)=\min \left\{p_{i} \circ W_{1}(q), p_{i} \circ\right.$ $\left.F_{2}\left(q_{2} r_{2}\right)\right\}$ for all $q \in V_{1}, q_{2} r_{2} \in E_{2}$,

(iii) $p_{i} \circ\left(F_{1} \times F_{2}\right)\left(\left(q_{1}, m\right)\left(r_{1}, m\right)\right)=\min \left\{p_{i} \circ F_{1}\left(q_{1} r_{1}\right), p_{i} \circ\right.$ $\left.W_{2}(m)\right\}$ for all $m \in V_{2}, q_{1} r_{1} \in E_{1}$,

(iv) $p_{i} \circ\left(F_{1} \times F_{2}\right)\left(\left(q_{1}, q_{2}\right)\left(r_{1}, r_{2}\right)\right)=0$ for all $\left(q_{1}, q_{2}\right)\left(r_{1}, r_{2}\right) \in$ $\left(V_{1} \tilde{x} V_{2}\right)^{2}-E$.

Definition 3. Given two graphs $G_{1}, G_{2}$ their composition, $G_{1}\left[G_{2}\right]=\left(V_{1} \times V_{2}, W_{1}\left[W_{2}\right], F_{1}\left[F_{2}\right]\right)$, is defined as follows: for $i=1,2, \ldots, m$, we have

(i) $p_{i} \circ\left(W_{1}\left[W_{2}\right]\right)\left(q_{1}, q_{2}\right)=\min \left\{p_{i} \circ W_{1}\left(q_{1}\right), p_{i} \circ W_{2}\left(q_{2}\right)\right\}$ for all $\left(q_{1}, q_{2}\right) \in V_{1} \times V_{2}$,

(ii) $p_{i} \circ\left(F_{1}\left[F_{2}\right]\right)\left(\left(q, q_{2}\right)\left(q, r_{2}\right)\right)=\min \left\{p_{i} \circ W_{1}(q), p_{i} \circ\right.$ $\left.F_{2}\left(q_{2} r_{2}\right)\right\}$ for all $q \in V_{1}, q_{2} r_{2} \in E_{2}$,

(iii) $p_{i} \circ\left(F_{1}\left[F_{2}\right]\right)\left(\left(q_{1}, m\right)\left(r_{1}, m\right)\right)=\min \left\{p_{i} \circ F_{1}\left(q_{1} r_{1}\right), p_{i} \circ\right.$ $\left.W_{2}(m)\right\}$ for all $m \in V_{2}, q_{1} r_{1} \in E_{1}$,

(iv) $p_{i} \circ\left(F_{1}\left[F_{2}\right]\right)\left(\left(q_{1}, q_{2}\right)\left(r_{1}, r_{2}\right)\right)=\min \left\{p_{i} \circ W_{2}\left(q_{2}\right), p_{i} \circ\right.$ $\left.W_{2}\left(r_{2}\right), p_{i} \circ F_{1}\left(q_{1} r_{1}\right)\right\}$ for all $\left(q_{1}, q_{2}\right)\left(r_{1}, r_{2}\right) \in E^{0}-E$, where $E^{0}=E \cup\left\{\left(q_{1}, q_{2}\right)\left(r_{1}, r_{2}\right) \mid q_{1} r_{1} \in E_{1}, q_{2} \neq r_{2}\right\}$.

Definition 4. The normal product of $G_{1}$ and $G_{2}$ is defined as the $m$-polar fuzzy graph $G_{1} \bullet G_{2}=\left(V_{1} \times V_{2}, W_{1} \bullet W_{2}, F_{1} \bullet F_{2}\right)$ on $G^{*}=(V, E)$, where $E=\left\{\left(\left(q, q_{2}\right)\left(q, r_{2}\right)\right) \mid q \in V_{1}, q_{2} r_{2} \in\right.$ $\left.E_{2}\right\} \cup\left\{\left(q_{1}, m\right)\left(r_{1}, m\right) \mid q_{1} r_{1} \in E_{1}, m \in V_{2}\right\} \cup\left\{\left(q_{1}, q_{2}\right)\left(r_{1}, r_{2}\right) \mid\right.$ $\left.q_{1} r_{1} \in E_{1}, q_{2} r_{2} \in E_{2}\right\}$ such that for $i=1,2, \ldots, m$, we have

(i) $p_{i} \circ\left(W_{1} \bullet W_{2}\right)\left(q_{1}, q_{2}\right)=\min \left\{p_{i} \circ W_{1}\left(q_{1}\right), p_{i} \circ W_{2}\left(q_{2}\right)\right\}$ for all $\left(q_{1}, q_{2}\right) \in V_{1} \times V_{2}$,

(ii) $p_{i} \circ\left(F_{1} \cdot F_{2}\right)\left(\left(q, q_{2}\right)\left(q, r_{2}\right)\right)=\min \left\{p_{i} \circ W_{1}(q), p_{i} \circ\right.$ $\left.F_{2}\left(q_{2} r_{2}\right)\right\}$ for all $q \in V_{1}, q_{2} r_{2} \in E_{2}$,

(iii) $p_{i} \circ\left(F_{1} \bullet F_{2}\right)\left(\left(q_{1}, m\right)\left(r_{1}, m\right)\right)=\min \left\{p_{i} \circ F_{1}\left(q_{1} r_{1}\right), p_{i} \circ\right.$ $\left.W_{2}(m)\right\}$ for all $m \in V_{2}, q_{1} r_{1} \in E_{1}$,

(iv) $p_{i} \circ\left(F_{1} \bullet F_{2}\right)\left(\left(q_{1}, q_{2}\right)\left(r_{1}, r_{2}\right)\right)=\min \left\{p_{i} \circ F_{1}\left(q_{1} r_{1}\right), p_{i} \circ\right.$ $\left.F_{2}\left(q_{2} r_{2}\right)\right\}$ for all $q_{1} q_{2} \in E_{1}$ and $r_{1} r_{2} \in E_{2}$.

Definition 5. The tensor product of $G_{1}$ and $G_{2}$ is defined as $G_{1} \otimes G_{2}=\left(V_{1} \times V_{2}, W_{1} \otimes W_{2}, F_{1} \otimes F_{2}\right)$ on $G^{*}=(V, E)$, where $E=\left\{\left(\left(q_{1}, q_{2}\right)\left(r_{1}, r_{2}\right)\right) \mid q_{1} r_{1} \in E_{1}, q_{2} r_{2} \in E_{2}\right\}$ such that for $i=1,2, \ldots, m$, we have

(i) $p_{i} \circ\left(W_{1} \otimes W_{2}\right)\left(q_{1}, q_{2}\right)=\min \left\{p_{i} \circ W_{1}\left(q_{1}\right), p_{i} \circ W_{2}\left(q_{2}\right)\right\}$ for all $\left(q_{1}, q_{2}\right) \in V_{1} \times V_{2}$,

(ii) $p_{i} \circ\left(F_{1} \otimes F_{2}\right)\left(\left(q_{1}, q_{2}\right)\left(r_{1}, r_{2}\right)\right)=\min \left\{p_{i} \circ F_{1}\left(q_{1} r_{1}\right), p_{i} \circ\right.$ $\left.F_{2}\left(q_{2} r_{2}\right)\right\}$ for all $q_{1} q_{2} \in E_{1}$ and $r_{1} r_{2} \in E_{2}$.

Definition 6. The union $G_{1} \cup G_{2}=\left(V_{1} \cup V_{2}, W_{1} \cup W_{2}, F_{1} \cup F_{2}\right)$ of the graphs $G_{1}=\left(V_{1}, W_{1}, F_{1}\right)$ and $G_{2}=\left(V_{2}, W_{2}, F_{2}\right)$ of $G_{1}{ }^{*}$ and $G_{2}{ }^{*}$, respectively, is defined as follows: for $i=1,2, \ldots, m$, we have (i)

$$
\begin{aligned}
p_{i} \circ\left(W_{1} \cup W_{2}\right)(q) & \begin{cases}p_{i} \circ W_{1}(q) & \text { if } q \in V_{1}-V_{2} \\
p_{i} \circ W_{2}(q) & \text { if } q \in V_{2}-V_{1} \\
\max \left\{p_{i} \circ W_{1}(q), p_{i} \circ W_{2}(q)\right\} & \text { if } q \in V_{1} \cap V_{2} .\end{cases}
\end{aligned}
$$

(ii)

$$
\begin{aligned}
& p_{i} \circ\left(F_{1} \cup F_{2}\right)(q r) \\
& = \begin{cases}p_{i} \circ F_{1}(q r) & \text { if } q r \in E_{1}-E_{2} \\
p_{i} \circ F_{2}(q r) & \text { if } q r \in E_{2}-E_{1} \\
\max \left\{p_{i} \circ F_{1}(q r), p_{i} \circ F_{2}(q r)\right\} & \text { if } q r \in E_{1} \cap E_{2} .\end{cases}
\end{aligned}
$$

$$
p_{i} \circ\left(F_{1} \cup F_{2}\right)(q r)=0 \quad \text { if } q r \in\left(V_{1} \tilde{\times} V_{2}\right)^{2}-E_{1} \cup E_{2} \text {. }
$$

Definition 7. The join of the graphs $G_{1}=\left(V_{1}, W_{1}, F_{1}\right)$ and $G_{2}=\left(V_{2}, W_{2}, F_{2}\right)$ of $G_{1}{ }^{*}$ and $G_{2}{ }^{*}$, respectively, is defined as $G_{1}+G_{2}=\left(V_{1} \cup V_{2}, W_{1}+W_{2}, F_{1}+F_{2}\right)$ such that for $i=1,2, \ldots, m$, we have the following:

(i) $p_{i} \circ\left(W_{1}+W_{2}\right)(q)=p_{i} \circ\left(W_{1} \cup W_{2}\right)(q)$ if $q \in V_{1} \cup V_{2}$.

(ii) $p_{i} \circ\left(F_{1}+F_{2}\right)(q r)=p_{i} \circ\left(F_{1} \cup F_{2}\right)(q r)$ if $q r \in E_{1} \cup E_{2}$.

(iii) $p_{i} \circ\left(F_{1}+F_{2}\right)(q r)=\min \left\{p_{i} \circ W_{1}(q), p_{i} \circ W_{2}(r)\right\}$ if $q r \epsilon$ $E^{\prime}$, where $E^{\prime}$ denotes the set of all edges joining the vertices of $V_{1}$ and $V_{2}$.

(iv) $p_{i} \circ\left(F_{1}+F_{2}\right)(q r)=0$ if $q r \in\left(V_{1} \tilde{\times} V_{2}\right)^{2}-E_{1} \cup E_{2} \cup E^{\prime}$.

\section{Degree of Vertices in $m$-Polar Fuzzy Graph}

Definition 8. Let $G=(V, W, F)$ be an $m$-polar fuzzy graph. Then the degree of a vertex $q$ in $G$ is defined as $d_{G}(q)=$ $\left\langle\sum_{q r \in E q \neq r} p_{1} \circ F(q r), \sum_{q r \in E q \neq r} p_{2} \circ F(q r), \sum_{q r \in E q \neq r} p_{3} \circ\right.$ $\left.F(q r), \ldots, \sum_{q r \in E} q \neq r p_{m} \circ F(q r)\right\rangle$.

Further, a highly irregular $m$-polar fuzzy graph is defined as an $m$-polar fuzzy graph $G=(V, W, F)$ in which every vertex of $G=(V, W, F)$ is adjacent to vertices with distinct degrees.

Example 9. Consider the graph $G=(V, W, F)$ of $G^{*}=(V$, $E)$, where $V=\{K, L, M, N\}, E=\{K L, L M, M N, N K\}$, $W=\{\langle 0.2,0.5,0.6\rangle / K,\langle 0.3,0.5,0.7\rangle / L,\langle 0.3,0.6,0.7\rangle / M,\langle 0.4$, $0.7,0.8\rangle / N\}$, and $F=\{\langle 0.2,0.4,0.5\rangle / K L,\langle 0.3,0.4,0.6\rangle / L M$, $\langle 0.3,0.5,0.6\rangle / M N,\langle 0.2,0.3,0.6\rangle / N K\}$ as in Figure 1.

In this graph, we have $d_{G}(K)=\langle 0.2,0.4,0.5\rangle+$ $\langle 0.2,0.3,0.6\rangle=\langle 0.4,0.7,1.1\rangle, d_{G}(L)=\langle 0.2,0.4,0.5\rangle+$ $\langle 0.3,0.4,0.6\rangle=\langle 0.5,0.8,1.1\rangle, d_{G}(M)=\langle 0.3,0.4,0.6\rangle+$ $\langle 0.3,0.5,0.6\rangle=\langle 0.6,0.9,1.2\rangle, d_{G}(N)=\langle 0.3,0.5,0.6\rangle+$ $\langle 0.2,0.3,0.6\rangle=\langle 0.5,0.8,1.2\rangle$. 


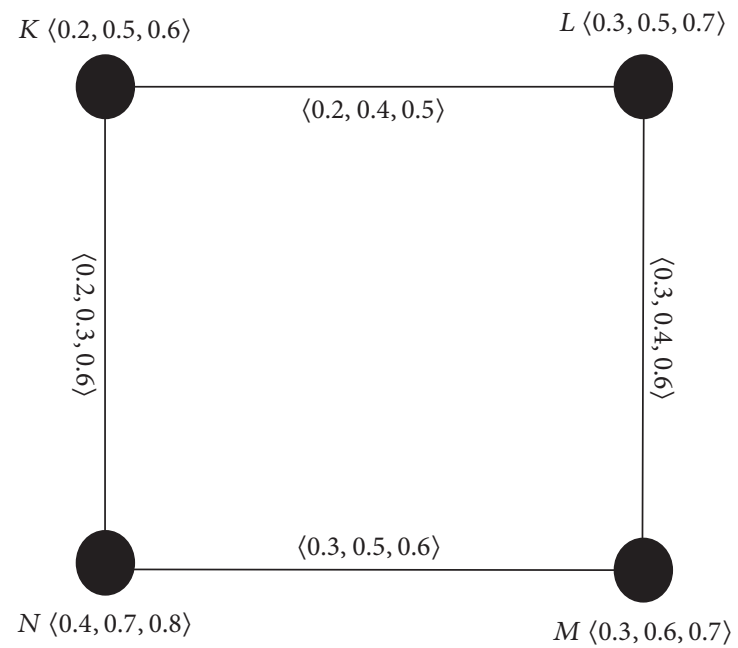

FIGURE 1: Highly irregular 3-polar fuzzy graph $G$.

\section{Cartesian Product and Vertex Degree}

From the definition of Cartesian product graphs, for every vertex $\left(q_{1}, q_{2}\right) \in V_{1} \times V_{2}$, we have

$$
\begin{aligned}
& d_{G_{1} \times G_{2}}\left(q_{1}, q_{2}\right) \\
& =\sum_{\left(q_{1}, q_{2}\right)\left(r_{1}, r_{2}\right) \in E} p_{i} \circ\left(F_{1} \times F_{2}\right)\left(\left(q_{1}, q_{2}\right)\left(r_{1}, r_{2}\right)\right) \\
& =\sum_{q_{1}=r_{1}, q_{2} r_{2} \in E_{2}} \min \left\{p_{i} \circ W_{1}\left(q_{1}\right), p_{i} \circ F_{2}\left(q_{2} r_{2}\right)\right\} \\
& \quad+\sum_{q_{2}=r_{2}, q_{1} r_{1} \in E_{1}} \min \left\{p_{i} \circ W_{2}\left(q_{2}\right), p_{i} \circ F_{1}\left(q_{1} r_{1}\right)\right\} \\
& \text { for } i=1,2, \ldots, m .
\end{aligned}
$$

Theorem 10. Let $G_{1}=\left(V_{1}, W_{1}, F_{1}\right)$ and $G_{2}=\left(V_{2}, W_{2}, F_{2}\right)$ be two graphs. If $p_{i} \circ W_{1} \geq p_{i} \circ F_{2}$ and $p_{i} \circ W_{2} \geq p_{i} \circ F_{1}$ then $d_{G_{1} \times G_{2}}\left(q_{1}, q_{2}\right)=d_{G_{1}}\left(q_{1}\right)+d_{G_{2}}\left(q_{2}\right)$ for $i=1,2, \ldots, m$.

Proof. By definition, we get

$$
\begin{aligned}
& d_{G_{1} \times G_{2}}\left(q_{1}, q_{2}\right) \\
& =\sum_{\left(q_{1}, q_{2}\right)\left(r_{1}, r_{2}\right)} p_{i} \circ\left(F_{1} \times F_{2}\right)\left(\left(q_{1}, q_{2}\right)\left(r_{1}, r_{2}\right)\right) \\
& =\sum_{q_{1}=r_{1}, q_{2} r_{2} \in E_{2}} \min \left\{p_{i} \circ W_{1}\left(q_{1}\right), p_{i} \circ F_{2}\left(q_{2} r_{2}\right)\right\} \\
& \quad+\sum_{q_{2}=r_{2}, q_{1} r_{1} \in E_{1}} \min \left\{p_{i} \circ W_{2}\left(q_{2}\right), p_{i} \circ F_{1}\left(q_{1} r_{1}\right)\right\} \\
& =\sum_{q_{2} r_{2} \in E_{2}} p_{i} \circ F_{2}\left(q_{2} r_{2}\right)+\sum_{q_{1} r_{1} \in E_{1}} p_{i} \circ F_{1}\left(q_{1} r_{1}\right) \\
& \quad\left(\text { Since } p_{i} \circ W_{1} \geq p_{i} \circ F_{2}, p_{i} \circ W_{2} \geq p_{i} \circ F_{1}\right) \\
& =d_{G_{1}}\left(q_{1}\right)+d_{G_{2}}\left(q_{2}\right) .
\end{aligned}
$$

Example 11. Consider two graphs $G_{1}, G_{2}$ and their Cartesian product, $G_{1} \times G_{2}$, as shown in Figure 2 .

Since $p_{i} \circ W_{1} \geq p_{i} \circ F_{2}$ and $p_{i} \circ W_{2} \geq p_{i} \circ F_{1}$, by Theorem 10, we have

$$
\begin{aligned}
d_{G_{1} \times G_{2}}\left(s_{1}, s_{2}\right) & =d_{G_{1}}\left(s_{1}\right)+d_{G_{2}}\left(s_{2}\right) \\
& =\langle 0.2,0.3,0.1\rangle+\langle 0.2,0.4,0.1\rangle \\
& =\langle 0.4,0.7,0.2\rangle, \\
d_{G_{1} \times G_{2}}\left(s_{1}, t_{2}\right) & =d_{G_{1}}\left(s_{1}\right)+d_{G_{2}}\left(t_{2}\right) \\
& =\langle 0.2,0.3,0.1\rangle+\langle 0.2,0.4,0.1\rangle \\
& =\langle 0.4,0.7,0.2\rangle \\
d_{G_{1} \times G_{2}}\left(t_{1}, s_{2}\right) & =d_{G_{1}}\left(t_{1}\right)+d_{G_{2}}\left(s_{2}\right) \\
& =\langle 0.2,0.3,0.1\rangle+\langle 0.2,0.4,0.1\rangle \\
& =\langle 0.4,0.7,0.2\rangle, \\
d_{G_{1} \times G_{2}}\left(t_{1}, t_{2}\right) & =d_{G_{1}}\left(t_{1}\right)+d_{G_{2}}\left(t_{2}\right) \\
& =\langle 0.2,0.3,0.1\rangle+\langle 0.2,0.4,0.1\rangle \\
& =\langle 0.4,0.7,0.2\rangle .
\end{aligned}
$$

\section{Composition Graph and Vertex Degree}

From the definition of composition graphs, for every vertex $\left(q_{1}, q_{2}\right) \in V_{1} \times V_{2}$, we have

$$
\begin{gathered}
d_{G_{1}\left[G_{2}\right]}\left(q_{1}, q_{2}\right)=\sum_{\left(q_{1}, q_{2}\right)\left(r_{1}, r_{2}\right) \in E} p_{i} \circ\left(F_{1}\left[F_{2}\right]\right)\left(\left(q_{1}, q_{2}\right)\right. \\
\left.\cdot\left(r_{1}, r_{2}\right)\right)=\sum_{q_{1}=r_{1}, q_{2} r_{2} \in E_{2}} \min \left\{p_{i} \circ W_{1}\left(q_{1}\right), p_{i}\right. \\
\left.\circ F_{2}\left(q_{2} r_{2}\right)\right\}+\sum_{q_{2}=r_{2}, q_{1} r_{1} \in E_{1}} \min \left\{p_{i} \circ W_{2}\left(q_{2}\right), p_{i}\right. \\
\left.\circ F_{1}\left(q_{1} r_{1}\right)\right\}+\sum_{q_{2} \neq r_{2}, q_{1} r_{1} \in E_{1}} \min \left\{p_{i} \circ W_{2}\left(r_{2}\right), p_{i}\right. \\
\left.\circ W_{2}\left(q_{2}\right), p_{i} \circ F_{1}\left(q_{1} r_{1}\right)\right\} \quad \text { for } i=1,2, \ldots, m .
\end{gathered}
$$

Theorem 12. Let $G_{1}=\left(V_{1}, W_{1}, F_{1}\right)$ and $G_{2}=\left(V_{2}, W_{2}, F_{2}\right)$ be two graphs. If $p_{i} \circ W_{1} \geq p_{i} \circ F_{2}$ and $p_{i} \circ W_{2} \geq p_{i} \circ F_{1}$ then $d_{G_{1}\left[G_{2}\right]}\left(q_{1}, q_{2}\right)=\left|V_{2}\right| d_{G_{1}}\left(q_{1}\right)+d_{G_{2}}\left(q_{2}\right)$ for all $\left(q_{1}, q_{2}\right) \in V_{1} \times V_{2}$ for $i=1,2, \ldots, m$.

Proof. By definition, we get

$$
\begin{gathered}
d_{G_{1}\left[G_{2}\right]}\left(q_{1}, q_{2}\right)=\sum_{\left(q_{1}, q_{2}\right)\left(r_{1}, r_{2}\right)} p_{i} \circ\left(F_{1}\left[F_{2}\right]\right)\left(\left(q_{1}, q_{2}\right)\right. \\
\left.\cdot\left(r_{1}, r_{2}\right)\right)=\sum_{q_{1}=r_{1}, q_{2} r_{2} \in E_{2}} \min \left\{p_{i} \circ W_{1}\left(q_{1}\right), p_{i}\right. \\
\left.\circ F_{2}\left(q_{2} r_{2}\right)\right\}+\sum_{q_{2}=r_{2}, q_{1} r_{1} \in E_{1}} \min \left\{p_{i} \circ W_{2}\left(q_{2}\right), p_{i}\right.
\end{gathered}
$$




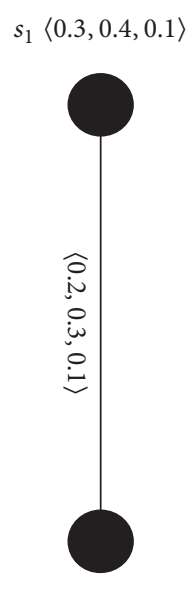

$t_{1}\langle 0.9,0.8,0.25\rangle$

$G_{1}$

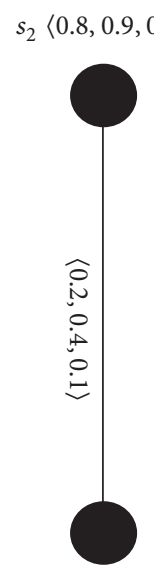

$t_{2}\langle 0.6,0.8,0.6\rangle$ $\left(s_{1}, s_{2}\right)\langle 0.3,0.4,0.1\rangle$

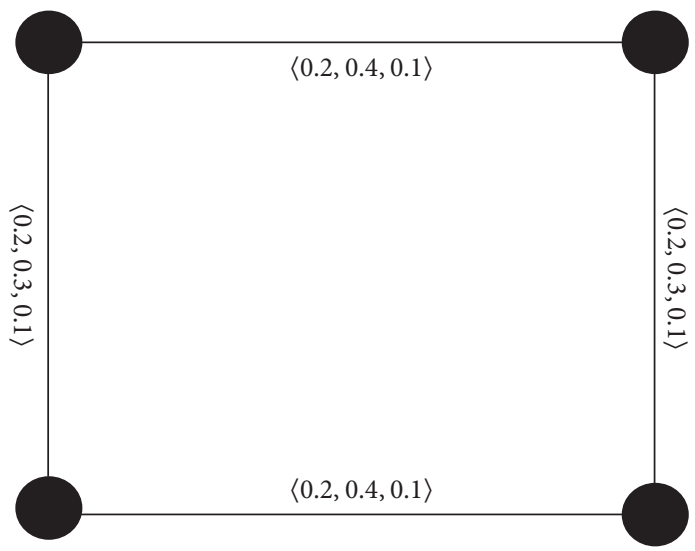

$\left(t_{1}, s_{2}\right)\langle 0.8,0.8,0.25\rangle$ $\left(t_{1}, t_{2}\right)\langle 0.6,0.8,0.25\rangle$

$G_{1} \times G_{2}$

FIgURE 2: Cartesian product of two 3-polar fuzzy graphs $G_{1}$ and $G_{2}$.

$s_{1}\langle 0.8,0.5,0.3\rangle$

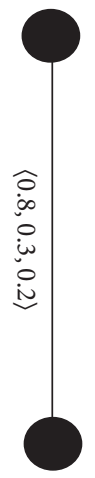

$t_{1}\langle 0.9,0.4,0.6\rangle$ $s_{2}\langle 0.8,0.6,0.2\rangle$

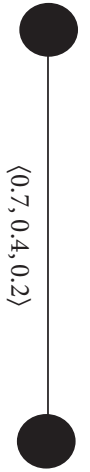

$t_{2}\langle 0.9,1.0,0.5\rangle$

$G_{2}$ $\left(s_{1}, s_{2}\right)\langle 0.8,0.5,0.2\rangle$

$\left(s_{1}, t_{2}\right)\langle 0.8,0.5,0.3\rangle$

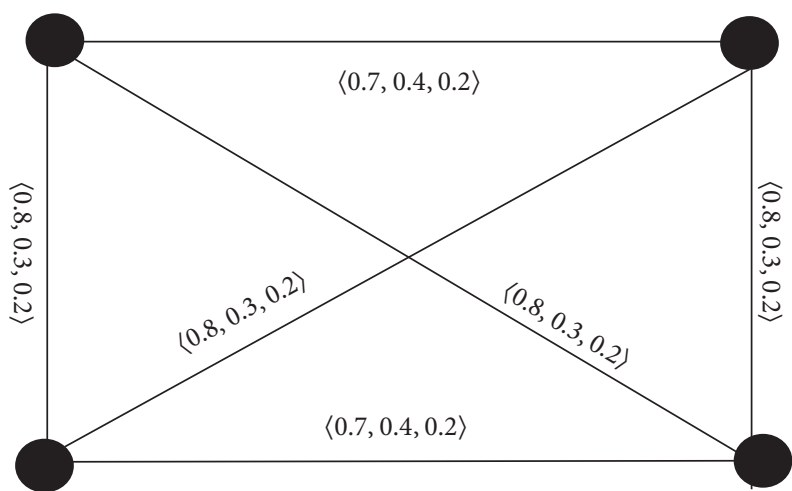

$\left(t_{1}, s_{2}\right)\langle 0.8,0.4,0.2\rangle$

$\left(t_{1}, t_{2}\right)\langle 0.9,0.4,0.5\rangle$

$G_{1}\left[G_{2}\right]$

FIGURE 3: Composition of two 3-polar fuzzy graphs $G_{1}$ and $G_{2}$.

$\left.\circ F_{1}\left(q_{1} r_{1}\right)\right\}+\sum_{q_{2} \neq r_{2}, q_{1} r_{1} \in E_{1}} \min \left\{p_{i} \circ W_{2}\left(r_{2}\right), p_{i}\right.$

$\left.\circ W_{2}\left(q_{2}\right), p_{i} \circ F_{1}\left(q_{1} r_{1}\right)\right\}=\sum_{q_{2} r_{2} \in E_{2}} p_{i} \circ F_{2}\left(q_{2} r_{2}\right)$

$+\sum_{q_{2}=r_{2}, q_{1} r_{1} \in E_{1}} p_{i} \circ F_{1}\left(q_{1} r_{1}\right)+\sum_{q_{2} \neq r_{2}, q_{1} r_{1} \in E_{1}} p_{i}$

$\circ F_{1}\left(q_{1} r_{1}\right)=d_{G_{2}}\left(q_{2}\right)+\left|V_{2}\right| \sum_{q_{1} r_{1} \in E_{1}} p_{i} \circ F_{1}\left(q_{1} r_{1}\right)$

$=\left|V_{2}\right| d_{G_{1}}\left(q_{1}\right)+d_{G_{2}}\left(q_{2}\right)$.
Example 13. Consider two graphs $G_{1}, G_{2}$ and their composition, $G_{1}\left[G_{2}\right]$, as shown in Figure 3.

Since $p_{i} \circ W_{1} \geq p_{i} \circ F_{2}$ and $p_{i} \circ W_{2} \geq p_{i} \circ F_{1}$, by Theorem 12, we have

$$
\begin{aligned}
d_{G_{1}\left[G_{2}\right]}\left(s_{1}, s_{2}\right) & =\left|V_{2}\right| d_{G_{1}}\left(s_{1}\right)+d_{G_{2}}\left(s_{2}\right) \\
& =2\langle 0.8,0.3,0.2\rangle+\langle 0.7,0.4,0.2\rangle \\
& =\langle 2.3,1.0,0.6\rangle, \\
d_{G_{1}\left[G_{2}\right]}\left(s_{1}, t_{2}\right) & =\left|V_{2}\right| d_{G_{1}}\left(s_{1}\right)+d_{G_{2}}\left(t_{2}\right) \\
& =2\langle 0.8,0.3,0.2\rangle+\langle 0.7,0.4,0.2\rangle \\
& =\langle 2.3,1.0,0.6\rangle,
\end{aligned}
$$




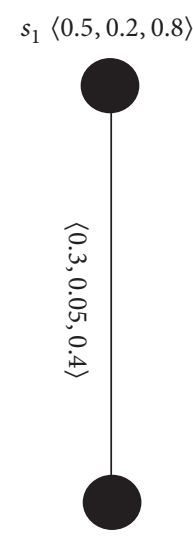

$t_{1}\langle 0.6,0.7,0.6\rangle$

$G_{1}$

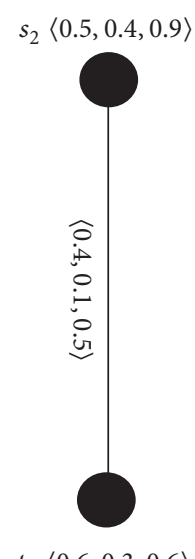

$t_{2}\langle 0.6,0.3,0.6\rangle$

$G_{2}$

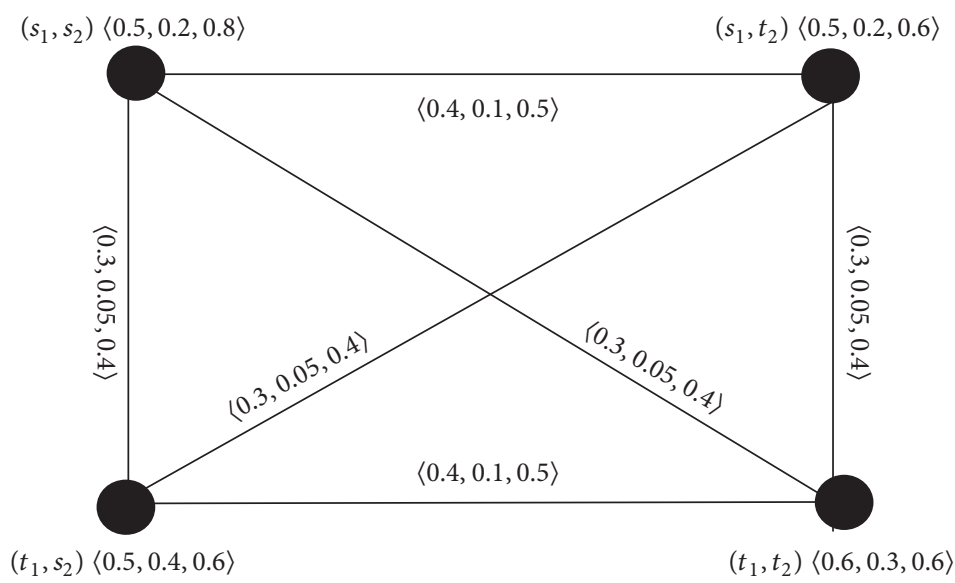

$G_{1} \cdot G_{2}$

FIGURE 4: Normal product of two 3-polar fuzzy graphs $G_{1}$ and $G_{2}$.

$$
\begin{aligned}
d_{G_{1}\left[G_{2}\right]}\left(t_{1}, s_{2}\right) & =\left|V_{2}\right| d_{G_{1}}\left(t_{1}\right)+d_{G_{2}}\left(s_{2}\right) \\
& =2\langle 0.8,0.3,0.2\rangle+\langle 0.7,0.4,0.2\rangle \\
& =\langle 2.3,1.0,0.6\rangle, \\
d_{G_{1}\left[G_{2}\right]}\left(t_{1}, t_{2}\right) & =\left|V_{2}\right| d_{G_{1}}\left(t_{1}\right)+d_{G_{2}}\left(t_{2}\right) \\
& =2\langle 0.8,0.3,0.2\rangle+\langle 0.7,0.4,0.2\rangle \\
& =\langle 2.3,1.0,0.6\rangle .
\end{aligned}
$$

\section{Normal Product and Vertex Degree}

From the definition of normal product graphs, for every vertex $\left(q_{1}, q_{2}\right) \in V_{1} \times V_{2}$, we have

$$
\begin{aligned}
& d_{G_{1} \cdot G_{2}}\left(q_{1}, q_{2}\right) \\
& =\sum_{\left(q_{1}, q_{2}\right)\left(r_{1}, r_{2}\right) \in E} p_{i} \circ\left(F_{1} \bullet F_{2}\right)\left(\left(q_{1}, r_{1}\right)\left(q_{2}, r_{2}\right)\right) \\
& =\sum_{q_{1}=r_{1}, q_{2} r_{2} \in E_{2}} \min \left\{p_{i} \circ W_{1}\left(q_{1}\right), p_{i} \circ F_{2}\left(q_{2} r_{2}\right)\right\} \\
& \quad+\sum_{q_{2}=r_{2}, q_{1} r_{1} \in E_{1}} \min \left\{p_{i} \circ W_{2}\left(q_{2}\right), p_{i} \circ F_{1}\left(q_{1} r_{1}\right)\right\} \\
& \quad+\sum_{q_{2} r_{2} \in E_{2}, q_{1} r_{1} \in E_{1}} \min \left\{p_{i} \circ F_{1}\left(q_{1} r_{1}\right), p_{i} \circ F_{2}\left(q_{2} r_{2}\right)\right\} \\
& \text { for } i=1,2, \ldots, m .
\end{aligned}
$$

Theorem 14. Let $G_{1}=\left(V_{1}, W_{1}, F_{1}\right)$ and $G_{2}=\left(V_{2}, W_{2}, F_{2}\right)$ be two graphs. If $p_{i} \circ W_{1} \geq p_{i} \circ F_{2}, p_{i} \circ W_{2} \geq p_{i} \circ F_{1}$ and $p_{i} \circ F_{1} \leq p_{i} \circ F_{2}$, then $d_{G_{1}} \circ G_{2}\left(q_{1}, q_{2}\right)=\left|V_{2}\right| d_{G_{1}}\left(q_{1}\right)+d_{G_{2}}\left(q_{2}\right)$ for $i=1,2, \ldots, m$.
Proof. By definition, we get

$$
\begin{aligned}
d_{G_{1}} \circ G_{2} & \left(q_{1}, q_{2}\right)=\sum_{\left(q_{1}, q_{2}\right)\left(r_{1}, r_{2}\right) \in E} p_{i} \circ\left(F_{1} \times F_{2}\right)\left(\left(q_{1}, r_{1}\right)\left(q_{2}, r_{2}\right)\right) \\
= & \sum_{q_{1}=r_{1}, q_{2} r_{2} \in E_{2}} \min \left\{p_{i} \circ W_{1}\left(q_{1}\right), p_{i} \circ F_{2}\left(q_{2} r_{2}\right)\right\} \\
& +\sum_{q_{2}=r_{2}, q_{1} r_{1} \in E_{1}} \min \left\{p_{i} \circ W_{2}\left(q_{2}\right), p_{i} \circ F_{1}\left(q_{1} r_{1}\right)\right\} \\
& +\sum_{q_{2} r_{2} \in E_{2}, q_{1} r_{1} \in E_{1}} \min \left\{p_{i} \circ F_{1}\left(q_{1} r_{1}\right), p_{i} \circ F_{2}\left(q_{2} r_{2}\right)\right\} \\
= & \sum_{q_{2} r_{2} \in E_{2}} p_{i} \circ F_{2}\left(q_{2} r_{2}\right)+\sum_{q_{2}=r_{2}, q_{1} r_{1} \in E_{1}} p_{i} \circ F_{1}\left(q_{1} r_{1}\right) \\
& +\sum_{q_{1} r_{1} \in E_{1}} p_{i} \circ F_{1}\left(q_{1} r_{1}\right)
\end{aligned}
$$

(Since $p_{i} \circ W_{1} \geq p_{i} \circ F_{2}, p_{i} \circ W_{2} \geq p_{i} \circ F_{1}, p_{i} \circ F_{1} \leq p_{i} \circ F_{2}$ )

$$
=\left|V_{2}\right| d_{G_{1}}\left(q_{1}\right)+d_{G_{2}}\left(q_{2}\right) \text {. }
$$

Example 15. Consider two graphs $G_{1}, G_{2}$ and their normal product, $G_{1} \bullet G_{2}$, is shown in Figure 4 .

Since $p_{i} \circ W_{1} \geq p_{i} \circ F_{2} p_{i} \circ W_{2} \geq p_{i} \circ F_{1}$ and $p_{i} \circ F_{1} \leq p_{i} \circ F_{2}$, by Theorem 14, we have

$$
\begin{aligned}
d_{G_{1} \bullet G_{2}}\left(s_{1}, s_{2}\right) & =\left|V_{2}\right| d_{G_{1}}\left(s_{1}\right)+d_{G_{2}}\left(s_{2}\right) \\
& =2\langle 0.3,0.05,0.4\rangle+\langle 0.4,0.1,0.5\rangle \\
& =\langle 1.0,0.2,1.3\rangle, \\
d_{G_{1} \bullet G_{2}}\left(s_{1}, t_{2}\right) & =\left|V_{2}\right| d_{G_{1}}\left(s_{1}\right)+d_{G_{2}}\left(t_{2}\right) \\
& =2\langle 0.3,0.05,0.4\rangle+\langle 0.4,0.1,0.5\rangle \\
& =\langle 1.0,0.2,1.3\rangle
\end{aligned}
$$




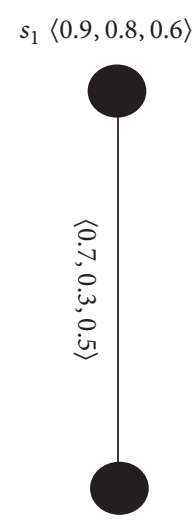

$t_{1}\langle 0.8,0.4,0.5\rangle$

$G_{1}$

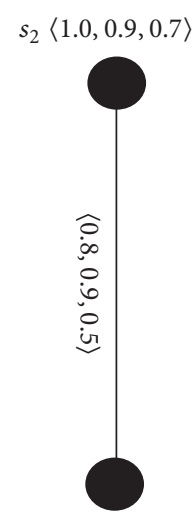

$t_{2}\langle 0.9,1.0,0.6\rangle$

$G_{2}$

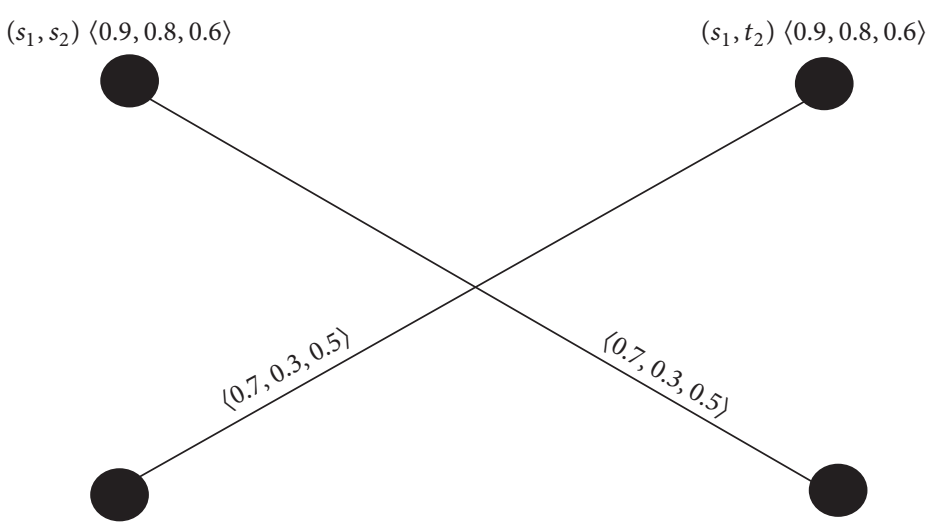

$\left(t_{1}, s_{2}\right)\langle 0.8,0.4,0.5\rangle$

$G_{1} \otimes G_{2}$ $\left(t_{1}, t_{2}\right)\langle 0.8,0.4,0.5\rangle$

Figure 5: Tensor product of two 3-polar fuzzy graphs $G_{1}$ and $G_{2}$.

$$
\begin{aligned}
d_{G_{1} \bullet G_{2}}\left(t_{1}, s_{2}\right) & =\left|V_{2}\right| d_{G_{1}}\left(t_{1}\right)+d_{G_{2}}\left(s_{2}\right) \\
& =2\langle 0.3,0.05,0.4\rangle+\langle 0.4,0.1,0.5\rangle \\
& =\langle 1.0,0.2,1.3\rangle, \\
d_{G_{1} \bullet G_{2}}\left(t_{1}, t_{2}\right) & =\left|V_{2}\right| d_{G_{1}}\left(t_{1}\right)+d_{G_{2}}\left(t_{2}\right) \\
& =2\langle 0.3,0.05,0.4\rangle+\langle 0.4,0.1,0.5\rangle \\
& =\langle 1.0,0.2,1.3\rangle .
\end{aligned}
$$

\section{Tensor Product and Vertex Degree}

From the definition of tensor product, for every vertex $\left(q_{1}, q_{2}\right) \in V_{1} \times V_{2}$ for $i=1,2, \ldots, m$

$$
\begin{gathered}
d_{G_{1} \otimes G_{2}}\left(q_{1}, q_{2}\right)=\sum p_{i} \circ\left(F_{1} \otimes F_{2}\right)\left(\left(q_{1}, q_{2}\right)\left(r_{1}, r_{2}\right)\right) \\
=\sum_{q_{1} r_{1} \in E_{1}} \min \left\{p_{i} \circ F_{1}\left(q_{1} r_{1}\right), p_{i} \circ F_{2}\left(q_{2} r_{2}\right)\right\} .
\end{gathered}
$$

Theorem 16. Let $G_{1}=\left(V_{1}, W_{1}, F_{1}\right)$ and $G_{2}=\left(V_{2}, W_{2}, F_{2}\right)$ be two m-polar fuzzy graphs. If $p_{i} \circ F_{2} \geq p_{i} \circ F_{1}$, then $d_{G_{1} \otimes G_{2}}\left(q_{1}, q_{2}\right)=d_{G_{1}}\left(q_{1}\right)$ and if $p_{i} \circ F_{1} \geq p_{i} \circ F_{2}$, then $d_{G_{1} \otimes G_{2}}\left(q_{1}, q_{2}\right)=d_{G_{2}}\left(q_{2}\right)$ for $i=1,2, \ldots, m$.

Proof. Let $p_{i} \circ F_{2} \geq p_{i} \circ F_{1}$. Then we get

$$
\begin{aligned}
& d_{G_{1} \otimes G_{2}}\left(q_{1}, q_{2}\right) \\
& \quad=\sum_{q_{1} r_{1} \in E_{1}} \min \left\{p_{i} \circ F_{1}\left(q_{1} r_{1}\right), p_{i} \circ F_{2}\left(q_{2} r_{2}\right)\right\} \\
& \quad=\sum p_{i} \circ F_{1}\left(q_{1} r_{1}\right)=d_{G_{1}}\left(q_{1}\right) .
\end{aligned}
$$

So, $d_{G_{1} \otimes G_{2}}\left(q_{1}, q_{2}\right)=d_{G_{1}}\left(q_{1}\right)$.
Let $p_{i} \circ F_{1} \geq p_{i} \circ F_{2}$. Then we get

$$
\begin{aligned}
& d_{G_{1} \otimes G_{2}}\left(q_{1}, q_{2}\right) \\
& \quad=\sum_{q_{1} r_{1} \in E_{1}} \min \left\{p_{i} \circ F_{1}\left(q_{1} r_{1}\right), p_{i} \circ F_{2}\left(q_{2} r_{2}\right)\right\} \\
& \quad=\sum p_{i} \circ F_{2}\left(q_{2} r_{2}\right)=d_{G_{2}}\left(q_{2}\right) .
\end{aligned}
$$

$$
\text { So, } d_{G_{1} \otimes G_{2}}\left(q_{1}, q_{2}\right)=d_{G_{2}}\left(q_{2}\right) \text {. }
$$

Example 17. Consider two graphs $G_{1}, G_{2}$ and their tensor product, $G_{1} \otimes G_{2}$, is shown in Figure 5 .

Since $p_{i} \circ F_{2} \geq p_{i} \circ F_{1}$, by Theorem 16 , we have

$$
\begin{aligned}
& d_{G_{1} \otimes G_{2}}\left(s_{1}, s_{2}\right)=d_{G_{1}}\left(s_{1}\right)=\langle 0.7,0.3,0.5\rangle, \\
& d_{G_{1} \otimes G_{2}}\left(s_{1}, t_{2}\right)=d_{G_{1}}\left(s_{1}\right)=\langle 0.7,0.3,0.5\rangle, \\
& d_{G_{1} \otimes G_{2}}\left(t_{1}, s_{2}\right)=d_{G_{1}}\left(t_{1}\right)=\langle 0.7,0.3,0.5\rangle, \\
& d_{G_{1} \otimes G_{2}}\left(t_{1}, t_{2}\right)=d_{G_{1}}\left(t_{1}\right)=\langle 0.7,0.3,0.5\rangle .
\end{aligned}
$$

\section{8. $\mu$-Complement of an $m$-Polar Fuzzy Graph}

Now for an $m$-polar fuzzy graph $G$, we introduce the notion of its $\mu$-complement.

Definition 18. The complement of an $m$-polar fuzzy graph $G=(V, W, F)$ is also an $m$-polar fuzzy graph $\bar{G}=(V, \bar{W}, \bar{F})$, where $\bar{W}=W$ and $\bar{F}$ is defined as follows:

$$
\begin{array}{r}
p_{i} \circ \bar{F}(q r)=\wedge\left(p_{i} \circ W(q), p_{i} \circ W(r)\right)-p_{i} \circ F(q r) \\
\forall q, r \in V \text { for } i=1,2, \ldots, m .
\end{array}
$$

Definition 19. Let $G=(V, W, F)$ be an $m$-polar fuzzy graph. If $G$ is isomorphic to $\bar{G}$ we say $G$ is self-complementary. Similarly, if $G$ is weak isomorphic to $\bar{G}$ then we say $G$ is selfweak complementary. 
Definition 20. The $\mu$-complement of $G$ is defined as $G^{\mu}=\left(V, W^{\mu}, F^{\mu}\right)$, where $W^{\mu}=W$ and $F^{\mu}$ is given by

$$
p_{i} \circ F^{\mu}(q r)= \begin{cases}\min \left\{p_{i} \circ W(q), p_{i} \circ W(r)\right\}-p_{i} \circ F(q r) & \text { if } p_{i} \circ F(q r)>0 \\ 0 & \text { if } p_{i} \circ F(q r)=0\end{cases}
$$

for $i=1,2, \ldots, m$.

Theorem 21. Let $G=(V, W, F)$ be a self-weak complementary and highly irregular graph. Then, we have $\sum_{q \neq r} p_{i} \circ F(q r) \leq$ $0.5 \sum_{q \neq r} \wedge\left\{p_{i} \circ W(q), p_{i} \circ W(r)\right\}$.

Proof. Let $G=(V, W, F)$ be a self-weak complementary, highly irregular graph of $G^{*}=(V, E)$. Then, there exists a weak isomorphism $g$ from $G$ to $\bar{G}$ such that for all $q, r \in V$, we get $p_{i} \circ W(q)=p_{i} \circ \bar{W}(g(q))=p_{i} \circ W(g(q)), p_{i} \circ F(q r) \leq$ $p_{i} \circ \bar{F}(g(q) g(r))$. From the definition of complement, from the above inequality, for all $q, r \in V$, we get that $p_{i} \circ F(q r) \leq$ $p_{i} \circ \bar{F}(g(q) g(r))=\wedge\left(p_{i} \circ W(q), p_{i} \circ W(r)\right)-p_{i} \circ F(g(q) g(r))$ and $p_{i} \circ F(q r)+p_{i} \circ F(g(q) g(r)) \leq \wedge\left(p_{i} \circ W(q), p_{i} \circ W(r)\right)$. Hence, $\sum_{q \neq r} p_{i} \circ F(q r)+\sum_{q \neq r} p_{i} \circ F(g(q) g(r)) \leq \sum_{q \neq r} \wedge\left(p_{i} \circ\right.$ $\left.W(q), p_{i} \circ W(r)\right)$. Thus, we get

$$
\begin{aligned}
& 2 \sum_{q \neq r} p_{i} \circ F(q r) \leq \sum_{q \neq r} \wedge\left(p_{i} \circ W(q), p_{i} \circ W(r)\right) \\
& \text { or } \sum_{q \neq r} p_{i} \circ F(q r) \leq 0.5 \sum_{q \neq r} \wedge\left\{p_{i} \circ W(q), p_{i} \circ W(r)\right\} .
\end{aligned}
$$

Theorem 22. Let $G=(V, W, F)$ be a graph which is selfcomplementary and highly irregular. Then, we have $\sum_{q \neq r} p_{i} \circ$ $F(q r)=0.5 \sum_{q \neq r} \wedge\left\{p_{i} \circ W(q), p_{i} \circ W(r)\right\}$, for all $q r \in \widetilde{V}^{2}, i=$ $1,2, \ldots, m$.

Proof. Let $G=(V, W, F)$ be a self-complementary, highly irregular graph of $G^{*}=(V, E)$. Then, there exists an isomorphism $g$ from $G$ to $\bar{G}$ such that $p_{i} \circ W(q)=p_{i} \circ \bar{W}(q)$ for all $q \in V$ and $p_{i} \circ F(q r)=p_{i} \circ \bar{F}(g(q) g(r))$ for all $q r \in \widetilde{V}^{2}$.

Let $q r \in \widetilde{V}^{2}$. Then, for $i=1,2, \ldots, m$, we have

$$
\begin{aligned}
p_{i} \circ & \bar{F}(g(q) g(r)) \\
= & \wedge\left(p_{i} \circ W(g(q)), p_{i} \circ W(g(r))\right)-p_{i} \\
& \circ F(g(q) g(r)) ;
\end{aligned}
$$

that is, $p_{i} \circ F(q r)=\wedge\left(p_{i} \circ W(g(q)), p_{i} \circ W(g(r))\right)-p_{i} \circ$ $F(g(q) g(r))$. Therefore,

$$
\begin{aligned}
& \sum_{q \neq r} p_{i} \circ F(q r)+\sum_{q \neq r} p_{i} \circ F(g(q) g(r)) \\
& =\sum_{q \neq r} \wedge\left\{p_{i} \circ W(g(q)), p_{i} \circ W(g(r))\right\} \\
& =\sum_{q \neq r} \wedge\left\{p_{i} \circ W(q), p_{i} \circ W(r)\right\} \\
& 2 \sum_{q \neq r} p_{i} \circ F(q r)=\sum_{q \neq r} \wedge\left\{p_{i} \circ W(q), p_{i} \circ W(r)\right\}
\end{aligned}
$$

i.e. $\sum_{q \neq r} p_{i} \circ F(q r)=0.5 \sum_{q \neq r} \wedge\left\{p_{i} \circ W(q), p_{i} \circ W(r)\right\}$.

Theorem 23. Let $G_{1}=\left(V_{1}, W_{1}, F_{1}\right)$ and $G_{2}=\left(V_{2}, W_{2}, F_{2}\right)$ be two graphs. If $G_{1}$ and $G_{2}$ are isomorphic, then their $\mu$ complements, $G_{1}{ }^{\mu}$ and $G_{2}{ }^{\mu}$, are also isomorphic.

Proof. Let $G_{1} \cong G_{2}$ and let $g$ be an isomorphism from $G_{1}$ to $G_{2}$. Then for $i=1,2, \ldots, m$

$$
\begin{aligned}
& p_{i} \circ W_{1}(q)=p_{i} \circ W_{2}(g(q)), \quad \forall q \in V_{1}, \\
& p_{i} \circ F_{1}(q r)=p_{i} \circ F_{2}(g(q) g(r)), \quad \forall q r \in E_{1} .
\end{aligned}
$$

If $p_{i} \circ F_{1}(q r)>0$, then $p_{i} \circ F_{2}(g(q) g(r))>0$, and

$$
\begin{aligned}
p_{i} \circ F_{1}{ }^{\mu}(q r)= & \min \left\{p_{i} \circ W_{1}(q), p_{i} \circ W_{1}(r)\right\}-p_{i} \\
& \circ F_{1}(q r) \\
= & \min \left\{p_{i} \circ W_{2}(g(q)), p_{i} \circ W_{2}(g(r))\right\} \\
& -p_{i} \circ F_{2}(g(q) g(r)) \\
= & p_{i} \circ F_{2}{ }^{\mu}(g(q) g(r)) .
\end{aligned}
$$

If $p_{i} \circ F_{1}(q r)=0$, then $p_{i} \circ F_{2}(g(q) g(r))=0$, and $p_{i} \circ$ $F_{1}^{\mu}(q r)=0=p_{i} \circ F_{2}^{\mu}(g(q) g(r))$.

Thus, $p_{i} \circ F_{1}{ }^{\mu}(q r)=p_{i} \circ F_{2}{ }^{\mu}(g(q) g(r))$ for all $q r \in E_{1}$. Therefore, $g$ from $G_{1}{ }^{\mu}$ to $G_{2}{ }^{\mu}$ is an isomorphism; that is, $G_{1}{ }^{\mu} \cong$ $G_{2}{ }^{\mu}$.

Theorem 24. Let $G_{1}=\left(V_{1}, W_{1}, F_{1}\right)$ and $G_{2}=\left(V_{2}, W_{2}, F_{2}\right)$ be two graphs such that $V_{1} \cap V_{2}=\phi$. Then $\left(G_{1}+G_{2}\right)^{\mu} \cong G_{1}{ }^{\mu} \cup G_{2}{ }^{\mu}$.

Proof. Let $I: V_{1} \cup V_{2} \rightarrow V_{1} \cup V_{2}$ be the identity map. To show that $\left(G_{1}+G_{2}\right)^{\mu} \cong G_{1}{ }^{\mu} \cup G_{2}{ }^{\mu}$, it is enough to prove that 
$\left(p_{i} \circ W_{1}+p_{i} \circ W_{2}\right)^{\mu}(q)=p_{i} \circ W_{1}^{\mu}(q) \cup p_{i} \circ W_{2}^{\mu}(q)$ and $\left(p_{i} \circ\right.$ $\left.F_{1}+p_{i} \circ F_{2}\right)^{\mu}(q r)=p_{i} \circ F_{1}{ }^{\mu}(q r) \cup p_{i} \circ F_{2}{ }^{\mu}(q r)$ for all $q, r \in V$ and for $i=1,2, \ldots, m$. Then for any $q, r \in V$, we have

$$
\left(p_{i} \circ W_{1}+p_{i} \circ W_{2}\right)^{\mu}(q)= \begin{cases}p_{i} \circ W_{1}(q) & \text { if } q \in V_{1} \\ p_{i} \circ W_{2}(q) & \text { if } q \in V_{2}\end{cases}
$$

$$
\begin{aligned}
& =\left\{\begin{array}{l}
p_{i} \circ W_{1}^{\mu}(q) \text { if } q \in V_{1} \\
p_{i} \circ W_{2}^{\mu}(q) \text { if } q \in V_{2}
\end{array}\right. \\
& =\left(p_{i} \circ W_{1}^{\mu} \cup p_{i} \circ W_{2}^{\mu}\right)(q) .
\end{aligned}
$$

Moreover,

$$
\begin{aligned}
\left(p_{i} \circ F_{1}+p_{i} \circ F_{2}\right)^{\mu}(q r)= & \min \left\{\left(p_{i} \circ W_{1}+p_{i} \circ W_{2}\right)(q),\left(p_{i} \circ W_{1}+p_{i} \circ W_{2}\right)(r)\right\}-\left(p_{i} \circ F_{1}+p_{i} \circ F_{2}\right)(q r) \\
= & \begin{cases}p_{i} \circ W_{1}(q) \wedge p_{i} \circ W_{1}(r)-p_{i} \circ F_{1}(q r) & \text { if } q r \in E_{1} \\
p_{i} \circ W_{2}(q) \wedge p_{i} \circ W_{2}(r)-p_{i} \circ F_{2}(q r) & \text { if } q r \in E_{2} \\
p_{i} \circ W_{1}(q) \wedge p_{i} \circ W_{2}(r)-p_{i} \circ W_{1}(q) \wedge p_{i} \circ W_{2}(r) \quad \text { if } q r \in E^{\prime}\end{cases} \\
= & \begin{cases}p_{i} \circ F_{1}^{\mu}(q r) & \text { if } q r \in E_{1} \\
p_{i} \circ F_{2}{ }^{\mu}(q r) & \text { if } q r \in E_{2} \\
0 & \text { if } q r \in E^{\prime}\end{cases} \\
= & \left(p_{i} \circ F_{1}^{\mu} \cup p_{i} \circ F_{2}^{\mu}\right)(q r) .
\end{aligned}
$$

Hence, the result is proved.

Theorem 25. Let $G_{1}=\left(V_{1}, W_{1}, F_{1}\right)$ and $G_{2}=\left(V_{2}, W_{2}, F_{2}\right)$ be two graphs such that $V_{1} \cap V_{2}=\phi$. Then $\left(G_{1} \cup G_{2}\right)^{\mu} \cong G_{1}{ }^{\mu} \cup G_{2}{ }^{\mu}$.

Proof. We shall prove that the identity map $I: V_{1} \cup V_{2} \rightarrow$ $V_{1} \cup V_{2}$ is the required isomorphism. Then for any $i, 1 \leq i \leq m$ we have the following:

If $q \in V_{1},\left(p_{i} \circ W_{1} \cup p_{i} \circ W_{2}\right)^{\mu}(q)=\left(p_{i} \circ W_{1} \cup p_{i} \circ W_{2}\right)(q)=$ $p_{i} \circ W_{1}(q)=p_{i} \circ W_{1}{ }^{\mu}(q)=\left(p_{i} \circ W_{1}^{\mu} \cup p_{i} \circ W_{2}{ }^{\mu}\right)(q)$.

If $q \in V_{2}$, then $\left(p_{i} \circ W_{1} \cup p_{i} \circ W_{2}\right)^{\mu}(q)=\left(p_{i} \circ W_{1} \cup p_{i} \circ\right.$ $\left.W_{2}\right)(q)=p_{i} \circ W_{2}(q)=p_{i} \circ W_{2}{ }^{\mu}(q)=\left(p_{i} \circ W_{2}^{\mu} \cup p_{i} \circ\right.$ $\left.W_{2}^{\mu}\right)(q)$.

If $q r \in E_{1}$, then $\left(p_{i} \circ F_{1} \cup p_{i} \circ F_{2}\right)^{\mu}(q r)=p_{i} \circ W_{1}(q) \wedge$ $p_{i} \circ W_{1}(r)-p_{i} \circ F_{1}(q r)=p_{i} \circ F_{1}^{\mu}(q r)=\left(p_{i} \circ F_{1}{ }^{\mu} \cup p_{i} \circ\right.$ $\left.F_{2}^{\mu}\right)(q r)$.

If $q r \in E_{2}$, then $\left(p_{i} \circ F_{1} \cup p_{i} \circ F_{2}\right)^{\mu}(q r)=p_{i} \circ W_{2}(q) \wedge$ $p_{i} \circ W_{2}(r)-p_{i} \circ F_{2}(q r)=p_{i} \circ F_{2}{ }^{\mu}(q r)=\left(p_{i} \circ F_{1}{ }^{\mu} \cup p_{i} \circ\right.$ $\left.F_{2}{ }^{\mu}\right)(q r)$.

Hence, the result is proved.

\section{9. m-Polar Fuzzy Graphs and Their Applications}

One of the important applications of $m$-polar fuzzy graphs is guidance in decision-making. For example, let us consider a scenario where a college wants to select a principal. Let $W=\{$ Kasim, Leo, Modi, Nandhu $\}$ be the set of doctorates shortlisted and let $F=\{$ Krishna, Srinu, Mano, Ramesh $\}$ be the set of college management members. The management has to make a selection taking into account the set of qualities $Q$ $=\{$ management skills, attitude, patents, and research $\}$. Each member from set $F$ can assign a value between 0 and 1 to each element listed in the set $Q$ for each candidate mentioned in set $W$, such as $W(K)=\langle 0.3,0.4,0.6,0.2\rangle, W(L)=$ $\langle 0.9,0.5,0.7,0.3\rangle, W(M)=\langle 0.4,0.7,0.3,0.5\rangle$, and $W(N)=$ $\langle 0.3,0.6,0.7,0.6\rangle$. Then we have a 4 -polar fuzzy graph, in which each member gives his opinion to $\mathrm{w}$ that belongs to $W$ on the basis of his qualities. So $W(K), W(L), W(M)$, and $W(N)$ denote the degree of management skills, attitude, patents, and research of each person given by the members of college management and edges denote the identical qualities of two persons (see Figure 6).

The membership degree of the edge $K L$ indicates that Kasim is $30 \%$ suitable, Leo is $40 \%$ suitable, Modi is $60 \%$ suitable, and Nandhu is $20 \%$ suitable. Thus, the edge $K L$ specifies that Modi is the suitable person for the post of principal. In the same way, $L M$ specifies Leo, $M N$ specifies Leo, and $N K$ also specifies Leo. Hence, according to all, Leo has a higher value of desirability among the shortlisted candidates and should be appointed as the principal.

\section{Conclusions}

Numerous uses can be harnessed from the theory of a fuzzy graph in the areas of number theory, algebra, topology, operation research, and so on. Not only researchers but also the common man can benefit from $m$-polar fuzzy data. It solves the day to day problems that are faced in the society related to probabilistic data. The novel approach discussed here enables decision makers to formulate better- preferred option with the help of unique case pattern than the conventional fuzzy 


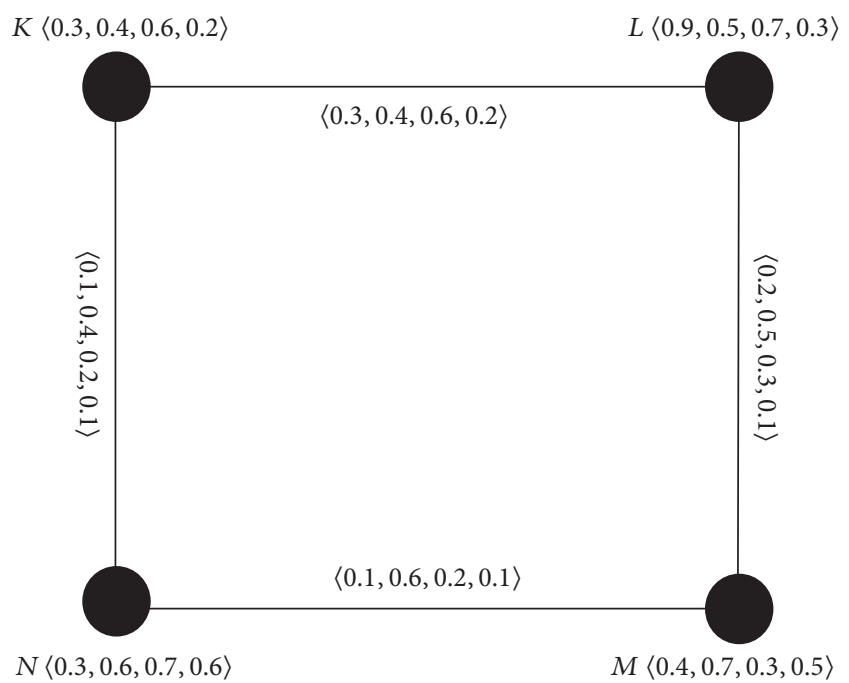

FIGURE 6: 4-polar fuzzy graph in decision-making.

graph approaches. The concepts complement, $\mu$-complement of an $m$-polar fuzzy graph, Cartesian product, composition, tensor product, and normal product are applied to $m$-polar fuzzy graphs and many results have been obtained.

\section{Conflicts of Interest}

The authors declare that there are no conflicts of interest regarding the publication of this paper.

\section{References}

[1] M. Akram, "Bipolar fuzzy graphs," Information Sciences. An International Journal, vol. 181, no. 24, pp. 5548-5564, 2011.

[2] K. R. Bhutani, "On automorphisms of fuzzy graphs," Pattern Recognition Letters, vol. 9, no. 3, pp. 159-162, 1989.

[3] J. Chen, S. Li, S. Ma, and X. Wang, "m-Polar fuzzy sets: an extension of bipolar fuzzy sets," The Scientific World Journal, vol. 2014, Article ID 416530, pp. 1-8, 2014.

[4] G. Ghorai and M. Pal, "Some properties of m-polar fuzzy graphs," Pacific Science Review A: Natural Science and Engineering, vol. 18, no. 1, pp. 38-46, 2016.

[5] J. N. Mordeson and C.-S. Peng, "Operations on fuzzy graphs," Information Sciences. An International Journal, vol. 79, no. 3-4, pp. 159-170, 1994.

[6] H. Rashmanlou, S. Samanta, M. Pal, and R. A. Borzooei, "Bipolar fuzzy graphs with categorical properties," International Journal of Computational Intelligence Systems, vol. 8, no. 5, pp. 808-818, 2015.

[7] M. S. Sunitha and A. Vijaya Kumar, "Complement of a fuzzy graph," Indian Journal of Pure and Applied Mathematics, vol. 33, no. 9, pp. 1451-1464, 2002.

[8] Ch. Ramprasad, P. L. N. Varma, N. Srinivasarao, and S. Satyanarayana, "Regular product m-polar fuzzy graphs and product m-polar fuzzy line graphs," Ponte, vol. 73, pp. 264-282, 2017. 

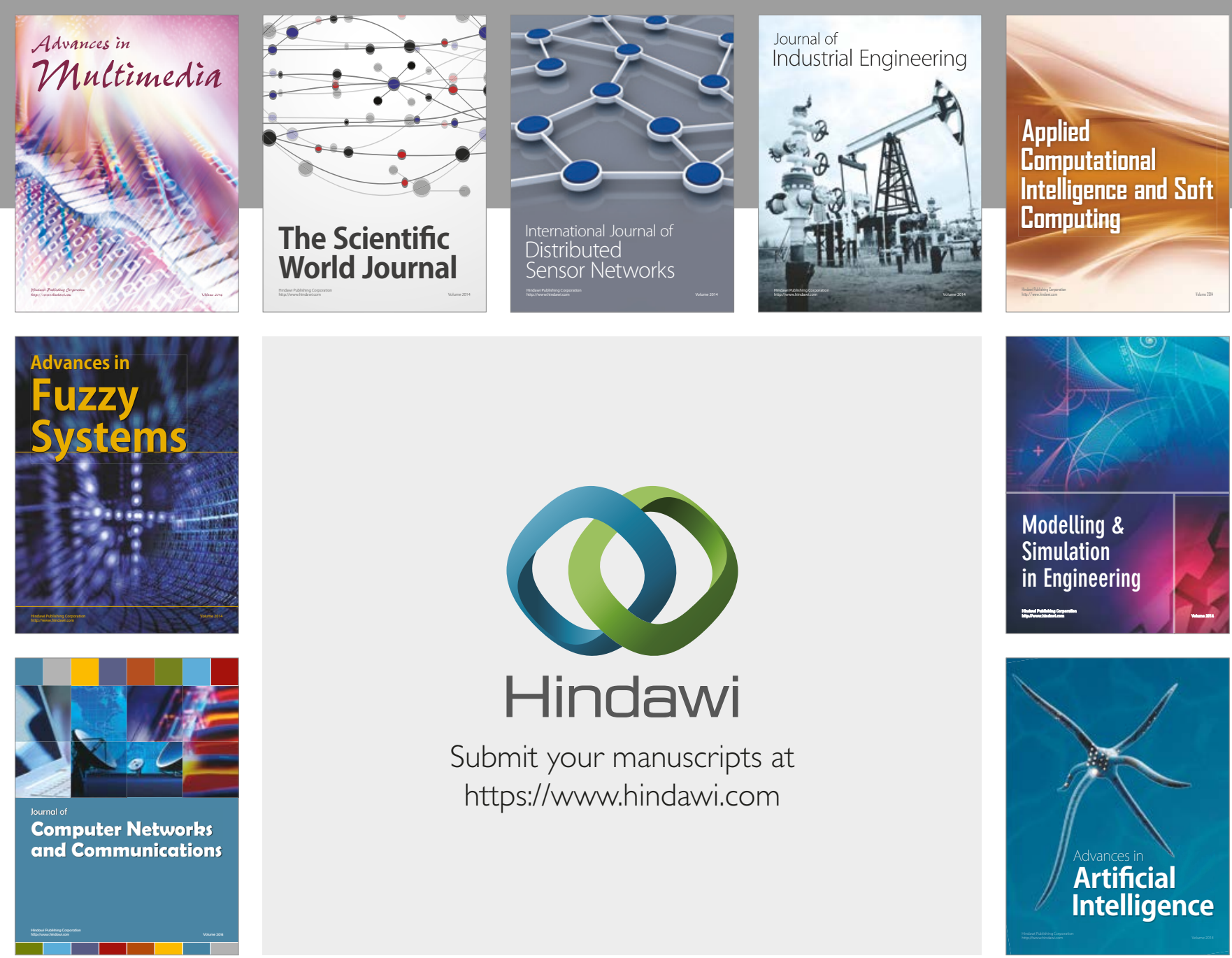

\section{Hindawi}

Submit your manuscripts at

https://www.hindawi.com
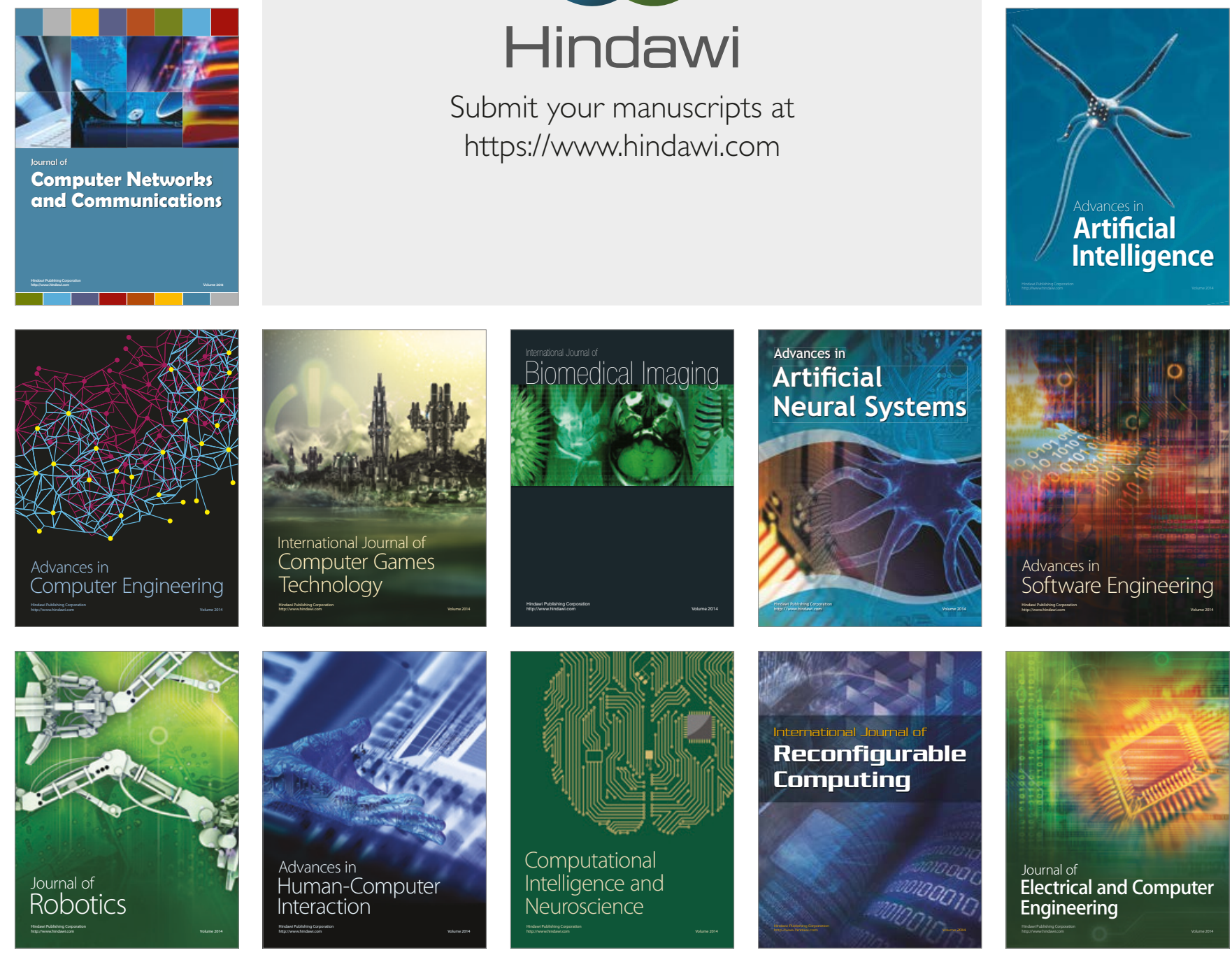